University of Wollongong

Research Online

Faculty of Engineering - Papers (Archive)

Faculty of Engineering and Information

Sciences

$1-1-2011$

\title{
Two-color terahertz response in bilayer graphene nanoribbons with spin- orbit coupling
}

Junfeng Liu

Peking University, Beijing, China

Bo Wang

Peking University Beijing, China

Zhongshui Ma

Peking University, Beijing, China, zma@uow.edu.au

Chao Zhang

University of Wollongong, czhang@uow.edu.au

Follow this and additional works at: https://ro.uow.edu.au/engpapers

Part of the Engineering Commons

https://ro.uow.edu.au/engpapers/4072

\section{Recommended Citation}

Liu, Junfeng; Wang, Bo; Ma, Zhongshui; and Zhang, Chao: Two-color terahertz response in bilayer graphene nanoribbons with spin-orbit coupling 2011, 061107-061107-3.

https://ro.uow.edu.au/engpapers/4072

Research Online is the open access institutional repository for the University of Wollongong. For further information contact the UOW Library: research-pubs@uow.edu.au 


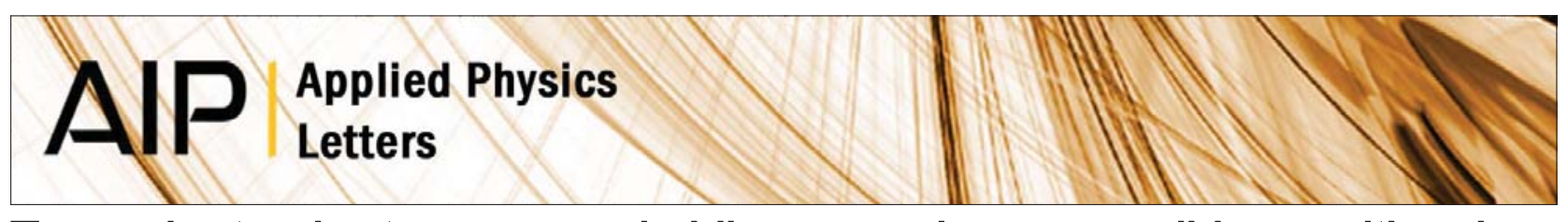

\section{Two-color terahertz response in bilayer graphene nanoribbons with spin- orbit coupling}

Junfeng Liu, Bo Wang, Zhongshui Ma, and C. Zhang

Citation: Appl. Phys. Lett. 98, 061107 (2011); doi: 10.1063/1.3555631

View online: http://dx.doi.org/10.1063/1.3555631

View Table of Contents: http://apl.aip.org/resource/1/APPLAB/v98/i6

Published by the American Institute of Physics.

\section{Related Articles}

Microwave and mechanical properties of quartz/graphene-based polymer nanocomposites Appl. Phys. Lett. 102, 072903 (2013)

Effects of Sr2+ or Sm3+ doping on electromagnetic and microwave absorption performance of LaMnO3 J. Appl. Phys. 113, 074903 (2013)

Autler-Townes splitting and quantum confined Stark effect of sideband peak in asymmetric double quantum wells J. Appl. Phys. 113, 043105 (2013)

Effects of relative density on microwave heating of various carbon powder compacts microwave-metallic multiparticle coupling using spatially separated magnetic fields

J. Appl. Phys. 113, 024902 (2013)

Terahertz time-domain measurement of non-Drude conductivity in silver nanowire thin films for transparent electrode applications

Appl. Phys. Lett. 102, 011109 (2013)

\section{Additional information on Appl. Phys. Lett.}

Journal Homepage: http://apl.aip.org/

Journal Information: http://apl.aip.org/about/about_the_journal

Top downloads: http://apl.aip.org/features/most_downloaded

Information for Authors: http://apl.aip.org/authors

\section{ADVERTISEMENT}

 Letters}



SURFACES AND INTERFACES

Focusing on physical, chemical, biological, structural, optical, magnetic and electrical and more...

\section{EXPLORE WHAT'S NEW IN APL}




\title{
Two-color terahertz response in bilayer graphene nanoribbons with spin-orbit coupling
}

\author{
Junfeng Liu, ${ }^{1, a)}$ Bo Wang, ${ }^{1}$ Zhongshui Ma, ${ }^{1}$ and C. Zhang ${ }^{2, b)}$ \\ ${ }^{1}$ School of Physics, Peking University, Beijing 100871, People's Republic of China \\ ${ }^{2}$ School of Engineering Physics, University of Wollongong, New South Wales 2522, Australia
}

(Received 12 January 2011; accepted 26 January 2011; published online 10 February 2011)

\begin{abstract}
We demonstrate that spin-orbit coupling can give rise to a strong terahertz response in metallic armchair bilayer graphene nanoribbons. The combination of the interlayer coupling and the spin-orbit coupling leads to double resonant optical response in the low frequency regime. The frequency separation of the two excitations is tunable with a gate voltage. (C) 2011 American Institute of Physics. [doi:10.1063/1.3555631]
\end{abstract}

Graphene exhibits many interesting properties not seen in any other systems. ${ }^{1,2}$ For example, the prediction and observation of electron-hole symmetry and a half-integer quantum Hall effect, ${ }^{3-5}$ finite conductivity at zero chargecarrier concentration, ${ }^{3}$ the strong suppression of weak localization, ${ }^{6-8}$ universal conductance, ${ }^{9-11}$ magnetic enhancement of optical conductance in graphene nanoribbons, ${ }^{12}$ and strong nonlinear response in the terahertz frequency regime. $^{13,14}$

Additional unique properties can be found in bilayer graphene (BLG). Chief among them is the so-called trigonal warping, ${ }^{15}$ a phenomenon solely due to the interlayer coupling. Electrons in bilayers can exhibits qualitatively different properties not seen in single layers, such as interlayer drags ${ }^{16}$ and correlations. ${ }^{17}$ The electronic and transport properties of BLG differ significantly from SLG in many respects, particularly at low energies in the "Dirac" regime. The electronic band structure of BLG has an asymmetry gap. ${ }^{18}$ In BLG the semiconductor gap can be controlled experimentally. ${ }^{19}$ The optical and magneto-optical far infrared properties of BLG has been studied. ${ }^{20}$ The role of the impurities in biased BLG has been clarified. ${ }^{21}$ One feature unique to $\mathrm{BLG}$ is the trigonal warping in the low energy dispersion. The minimum conductivity in BLG is six times as large as that for single layer graphene. ${ }^{15}$ This six time enhancement is independent of the strength of the trigonal warping, indicating the topological nature of the bilayer coupling.

In a recent work, ${ }^{22}$ it was shown that the optical response of graphene nanostructures can be significantly enhanced in a subclass of graphene nanoribbon. This subclass of nanoribbons is armchair ribbon of width $3 n-1$. In such ribbons, the lowest conduction band touches the highest valence band at the zone center while the next levels are only a 10-100 $\mathrm{meV}$ above. The transitions between the asymmetric bands are one to two orders of magnitude stronger than the universal conductance of infinite graphene.

In this paper, we shall show that for this class of armchair ribbon, the strong terahertz response can be further enhanced under a finite spin-orbit interaction (SOI). In fact, the SOI can be completely ignored in all ribbons other than

\footnotetext{
${ }^{a}$ Present address: Department of Physics and Materials Science, City University of Hong Kong, Hong Kong.

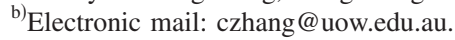

this subclass of armchair ribbon. SOI opens a small gap at the zone center. The electronic transition over the gap (valence 1 to conduction 1 , or $v 1-\mathrm{c} 1)$ is as strong as that of asymmetric transition (v1-c2 and v2-c1). As a result, there are two distinct excitations at the low energy regime. The v1-c1 transition is due to the SOI and the v1-c2 or v2-c1 transition is due the interlayer coupling. Together the ribbon exhibits the two-color excitations in the low energy regime.

A typical BLG sheet consists of two SLG layers stacked in the orientation shown in Fig. 1. Several forms of the Hamiltonian for BLG are used in the literature depending on the approximations used and the relative orientations of the two layers. The original consideration was given by Slonczewski-Weiss-McClure which included all three interlayer coupling terms. ${ }^{23,24}$ As usual, the nearest neighbor A-B and B-A coupling is included, which is given here by $t$ $=3.0 \mathrm{eV}$. The most prominent interlayer coupling term is the B1-A2 coupling between sites which are directly above (or below) each other. ${ }^{25,26}$ Here we denote this term as $\gamma_{1}$ $=0.12 t$. The second term is $\gamma_{3}=0.1 t, \gamma_{3}$ is the hopping energy between A1 and B2 which are not directly above or below each other. The other two interlayer coupling terms are the A1-A2 and B1-B2 coupling between inequivalent sites which are offset by an amount $a=1.42 \dot{A}$. These two coupling terms have the same strength $\gamma_{4}=0.04 t$. All energies will be normalized relative to the nearest neighbor hopping bandwidth $t$. The physical width of the ribbon are $W=(3 N / 2$ $-1) a$ for zigzag ribbon and $W=\sqrt{3} a / 2(N-1)$ for armchair ribbon.

The Hamiltonian of a BLG in the absence of spin-orbit couplings can be written as

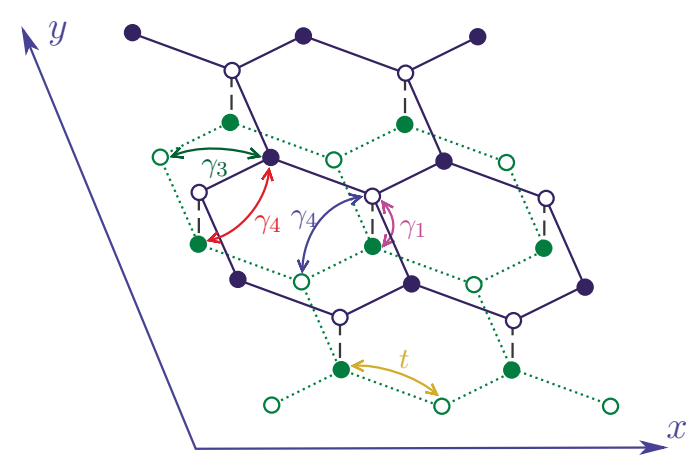

FIG. 1. (Color online) The BLGNRs showing various coupling parameters. 


$$
\begin{aligned}
& H_{\mathrm{BLG}}=t \sum_{l,\langle i j\rangle} a_{l, i}^{\dagger} b_{l, j}+\gamma_{1} \sum_{l, i} b_{1, i}^{\dagger} a_{2, i}+\gamma_{3} \sum_{\langle i j\rangle} a_{1, i}^{\dagger} b_{2, j}, \\
& +\gamma_{4} \sum_{\langle i j\rangle}\left(a_{1, i}^{\dagger} a_{2, j}+b_{1, i}^{\dagger} b_{2, j}\right)+H . C .
\end{aligned}
$$

where $a_{l, i}\left(b_{l, i}\right)$ is the annihilation operator at sublattice $\mathrm{A}(\mathrm{B})$, in the layer $l=1,2$, at site $R_{i}$.

The Rashba spin-orbit coupling term reads

$$
H_{\mathrm{RSOI}}=i \frac{\alpha}{a} \sum_{l,\langle i j\rangle}\left(a_{l, i}^{\dagger}\left[\sigma \times \mathbf{d}_{i j}\right]_{z} b_{l, j}+H . C .\right) \text {. }
$$

The total Hamiltonian for infinite BLG is $H=H_{\mathrm{BLG}}$ $+H_{\mathrm{RSOI}}$. Here $\mathbf{d}_{i j}$ is the displacement vector between the lattice sites $i$ and $j, \sigma$ is the Pauli matrices. Equation (3) is the extrinsic spin-orbit coupling. It couples only nearest neighbors with opposite spin. The intrinsic SOI opens a gap and gives rise to a quantum spin Hall phase due to localized edge states. $^{27}$ In general, the intrinsic spin-orbit coupling in graphene is weak, only a few microelectron volts. ${ }^{28}$ The extrinsic part can be regarded as a Rashba SOI. The presence of the extrinsic SO coupling is due to the breaking of the inversion symmetry of the graphene lattice. The coefficient $\alpha$ depends on the strength of the perpendicular electric field or the interaction with a substrate. ${ }^{29}$ Some papers reported that the Rashba coupling can be turned to $1 \mathrm{meV}$ for typical values of an external electric field $(50 \mathrm{~V} / 300 \mathrm{~nm})$. Breaking of the up-down symmetry by a substrate can result in an extrinsic spin-orbit coupling in the scale of $10 \mathrm{meV}$. The effect of impurities can increase the Rashba spin-orbit coupling to $7 \mathrm{meV}$. Therefore, the strength of extrinsic spin-orbit coupling is stronger than that of the intrinsic spin-orbit coupling by three orders of magnitude. For this reason, we shall focus our studies only on the effect of extrinsic spin-orbit coupling (which is also referred to as Rashba spin-orbit coupling). ${ }^{30}$

Now we construct an eigenvector space for ribbons where each eigenstate is denoted by longitudinal wave vector $k=k_{y}$ and an index $j(j=1,2,3 \ldots 8 N)$. And the eigenfunctions are $\psi_{k j}=\xi_{j} e^{i k y}$ which satisfy $H \psi_{k j}=\epsilon_{k j} \psi_{k j}$, where $\xi_{j}$ denote the $j$ th eigenvector of the Hamiltonian.

The energy band structure for armchair bilayer graphene nanoribbons (BLGNRs) are shown in Fig. 2. The SOI parameter is $\alpha=200 \mathrm{meV}$. The SOI induced band gap for an $N$ $=5$ ribbon and an $N=8$ ribbon is approximately same, but the gap for $N=8$ is a little smaller. This suggests that SOI induced gap decreases slowly with ribbon width and vanishes in the limit of infinite BLG. The other interesting effect is that the SOI lifts the spin degeneracy at the $\Gamma$ point. The two spin states are now separated in the $k_{y}$ direction by a distance of $0.1 \pi / 3 a$. The minimum direct gap for both spin state is now at $k_{y}= \pm 0.05 \pi / 3 a$.

In Fig. 3, we plot the dependence of the band gap as a function of SOI parameter for an $N=5$ armchair BLGNR under various interlayer coupling conditions. It is found that the interlayer coupling $\gamma_{3}$ plays the most dominant role for the SOI to be effective. We have multiplied the $\gamma_{3}=0$ curves by a factor of 30 or $10^{4}$ in order to see their $\alpha$-dependence. If $\gamma_{3}$ is zero, the SOI effect can be practically neglected. On the other hand if $\gamma_{3}$ is finite, the band gap grows linearly with the SOI parameter. The effect of $\gamma_{4}$ is not noticeable in the $\alpha$-dependence of the band gap.

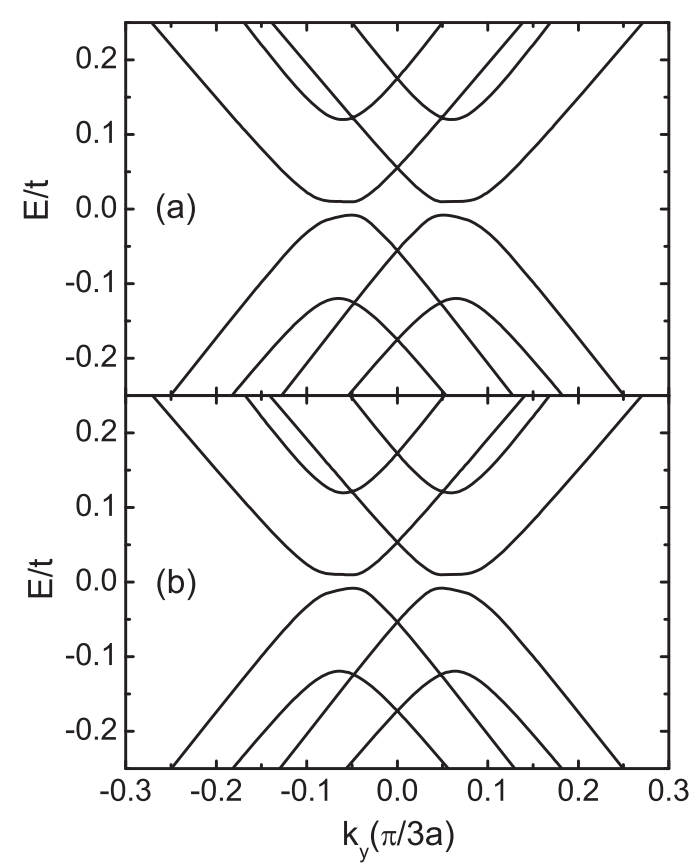

FIG. 2. Band structure of an $N=5$ (a) and an $N=8$ (b) armchair BLGNR, the SOI is $\alpha=200 \mathrm{meV}$. The curves with $\gamma_{3}=0$ have been multiplied by a large numerical factor to show their $\alpha$-dependence.

In order to calculate the conductance of ribbons using the Kubo formula, we first write the form of current operator

$$
J_{x}=c \frac{\partial H}{\partial A_{x}}, \quad J_{y}=e \frac{\partial H}{\partial\left(\hbar k_{y}\right)},
$$

where we must introduce an infinitesimal transversal vector potential $A_{x}$ and rewrite the Harper equation for solving $J_{x}$. By introducing the field operator

$$
\hat{\psi}(x, y)=\sum_{k, j} \xi_{j} e^{i k y} a_{k j},
$$

where $a_{k j}$ is the annihilation operator of the $j$ th eigenstate with wave vector $k=k_{y}$. The current operator can be expressed in the second quantization form

$$
\hat{J}_{\mu}=\int d y \hat{\psi}^{\dagger}(x, y) J_{\mu} \hat{\psi}(x, y)=\sum_{k j j^{\prime}} J_{j j^{\prime}}^{\mu} a_{k j}^{\dagger} a_{k j^{\prime}},
$$

with $J_{j j^{\prime}}^{\mu}=\xi_{j}^{\dagger} J_{\mu} \xi_{j^{\prime}}, \quad \mu=x, y$. According to Kubo formula, the ac conductance is found as

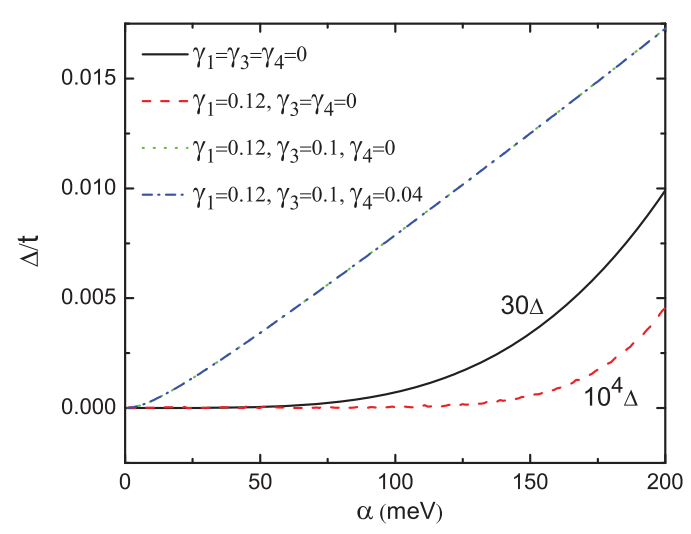

FIG. 3. (Color online) The dependence of the band gap of an $N=5$ armchair BLGNR on SOI parameter. 


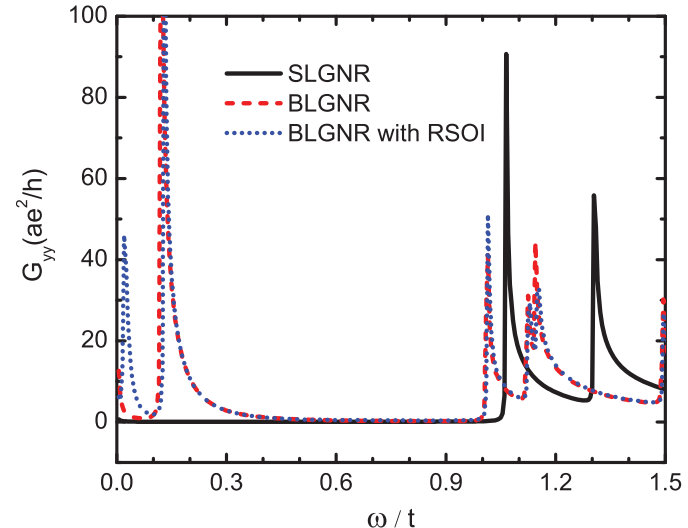

FIG. 4. (Color online) Optical conductance of an $N=8$ BLGNR with the SOI parameter $\alpha=0$ and $\alpha=200 \mathrm{meV}$. Also shown is the optical conductance of a single layer graphene nanoribbon.

$$
G_{\mu \nu}(\omega)=-\frac{1}{i \omega} \sum_{k j j^{\prime}} J_{j j^{\prime}}^{\mu} J_{j^{\prime} j}^{\nu} \frac{f_{k j}-f_{k j^{\prime}}}{\hbar \omega+\epsilon_{k j}-\epsilon_{k j^{\prime}}+i \delta},
$$

where $f_{k j}$ and $f_{k j^{\prime}}$ are Fermi distribution functions and $\delta$ is positive infinitesimal.

In Fig. 4, we plot the optical conductance versus frequency for an $N=8$ armchair BLGNR for a typical value of $\alpha=200 \mathrm{meV}$. In the absence of SOI, these is a excitation peak in the terahertz regime in metallic ribbons. This low energy peak can be one to two orders of magnitude stronger than the universal conductance. This strong optical response is due to asymmetric electronic transition (AET) mentioned earlier. Therefore, it has been proposed that the metallic armchair BLGNR can be the candidate for developing graphene based terahertz photonics. Under a finite SOI, an additional excitation emerges at the energy lower than the energy associated with the AET. The spectrum now has the twin peak optical response at very energies. While the peak position of AET for a given ribbon is fixed, both the intensity and position of SOI induced response peak can be tuned with a gate voltage, providing an excellent mechanism of two-color response in the terahertz frequency regime.

In conclusion, we have shown that the commonly neglected SOI in graphene can strongly affect the optical response of a subclass of BLGNR in the low frequency regime, resulting in a strong absorption peak at very low frequencies.

Z.S.M. acknowledge the financial support from NNSFC Grant Nos. 10874002 and 91021017. C.Z. acknowledges support from the Australian Research Council Grant No. DP0879151.

${ }^{1}$ K. S. Novoselov, A. K. Geim, S. V. Morozov, D. Jiang, Y. Zhang, S. V. Dubonos, I. V. Grigorieva, A. A. Firsov, Science 306, 666 (2004).

${ }^{2}$ A. K. Geim and K. S. Novoselov, Nature Mater. 6, 183 (2007).

${ }^{3}$ K. S. Novoselov, A. K. Geim, S. V. Morozov, D. Jiang, M. I. Katsnelson, I. V. Grigorieva, S. V. Dubonos, and A. A. Firsov, Nature (London) 438, 197 (2005)

${ }^{4}$ Y. Zhang, Y. W. Tan, H. L. Stormer, and P. Kim, Nature (London) 438, 201 (2005).

${ }^{5}$ C. Berger, Z. Song, X. Li, X. Wu, N. Brown, C. Naud, D. Mayou, T. Li, J. Hass, A. N. Marchenkov, E. H. Konrad, P. N. First, and W. A. de Heer, Science 312, 1191 (2006).

${ }^{6}$ H. Suzuura and T. Ando, Phys. Rev. Lett. 89, 266603 (2002); J. Phys. Soc. Jpn. 72, 69 (2003).

${ }^{7}$ S. V. Morozov, K. S. Novoselov, M. I. Katsnelson, F. Schedin, L. A. Ponomarenko, D. Jiang, and A. K. Geim, Phys. Rev. Lett. 97, 016801 (2006).

${ }^{8}$ D. V. Khveshchenko, Phys. Rev. Lett. 97, 036802 (2006).

${ }^{9}$ V. P. Gusynin, S. G. Sharapov, and J. P. Carbotte, Phys. Rev. Lett. 96, 256802 (2006).

${ }^{10}$ A. B. Kuzmenko, E. van Heumen, F. Carbone, and D. van der Marel, Phys. Rev. Lett. 100, 117401 (2008).

${ }^{11}$ R. R. Nair, P. Blake, A. N. Grigorenko, K. S. Novoselov, T. J. Booth, T. Stauber, N. M. R. Peres, and A. K. Geim, Science 320, 1308 (2008).

${ }^{12}$ J. Liu, A. R. Wright, C. Zhang, and Z. Ma, Appl. Phys. Lett. 93, 041106 (2008).

${ }^{13}$ S. A. Mikhailov and K. Ziegler, J. Phys.: Condens. Matter 20, 384204 (2008).

${ }^{14}$ A. R. Wright, X. G. Xu, J. C. Cao, and C. Zhang, Appl. Phys. Lett. 95, 072101 (2009).

${ }^{15}$ J. Cserti, A. Csordas, and G. David, Phys. Rev. Lett. 99, 066802 (2007).

${ }^{16}$ C. Zhang and Y. Takahashi, J. Phys.: Condens. Matter 5, 5009 (1993).

${ }^{17}$ C. Zhang, Phys. Rev. B 49, 2939 (1994).

${ }^{18}$ E. McCann, Phys. Rev. B 74, 161403(R) (2006).

${ }^{19}$ T. Ohta, A. Bostwick, T. Seyller, K. Horn, and E. Rotenberg, Science 313, 951 (2006).

${ }^{20}$ D. S. L. Abergel and V. I. Fal'ko, Phys. Rev. B 75, 155430 (2007).

${ }^{21}$ J. Nilsson and A. H. Castro Neto, Phys. Rev. Lett. 98, 126801 (2007).

${ }^{22}$ A. R. Wright, J. C. Cao, and C. Zhang, Phys. Rev. Lett. 103, 207401 (2009).

${ }^{23}$ J. W. McClure, Phys. Rev. 108, 612 (1957).

${ }^{24}$ J. C. Slonczewski and P. R. Weiss, Phys. Rev. 109, 272 (1958).

${ }^{25}$ D. D. L. Chung, J. Mater. Sci. 37, 1475 (2002).

${ }^{26}$ J. Nilsson, A. H. Castro Neto, F. Guinea, and N. M. R. Peres, Phys. Rev. B 78, 045405 (2008).

${ }^{27}$ C. L. Kane and M. J. Mele, Phys. Rev. Lett. 95, 226801 (2005).

${ }^{28}$ H. Min, J. E. Hill, N. A. Sinitsyn, B. R. Sahu, L. Kleinman, and A. H. MacDonald, Phys. Rev. B 74, 165310 (2006).

${ }^{29}$ C. Ertler, S. Konschuh, M. Gmitra, and J. Fabian, Phys. Rev. B 80, 041405 (2009).

${ }^{30}$ M. Zarea and N. Sandler, Phys. Rev. B 79, 165442 (2009). 